\title{
Current Trends in Use of Implantable Cardioverter Defibrillators and Cardiac Resynchronization Therapy With a Pacemaker or Defibrillator in Japanese Pediatric Patients - Results From a Nationwide Questionnaire Survey -
}

Tsugutoshi Suzuki, MD; Naokata Sumitomo, MD, PhD; Jun Yoshimoto, MD; Aya Miyazaki, MD; Kazuhiro Hinokiyama, MD, PhD; Hiroya Ushinohama, MD, PhD; Satoshi Yasukochi, MD

\begin{abstract}
Background: In Japan, the use of implantable cardioverter defibrillators (ICDs), cardiac resynchronization therapy with a biventricular pacemaker (CRTP) and CRT with a defibrillator (CRTD) in children has not been studied statistically, and dual-chamber (DDD) pacemakers are still used for pediatric CRT because of current government regulations.

Methods and Results: Data were obtained from 15 children's and 74 general hospitals through a questionnaire survey regarding the aforementioned therapies performed before 2012 in Japanese children ( $<16$ years old). ICD, CRT with DDD, CRTP and CRTD were used in $64(42 \%), 47(31 \%), 34(22 \%)$ and $7(5 \%)$, respectively, of all cases reported ( $n=152)$. Among all CRTP and CRT-DDD cases $(n=81)$, the use of DDD accounted for $41 \%$ in general hospitals vs. $89 \%$ in children's hospitals, and CRT-DDD and CRTP were effective in 67 cases (83\%). Of 64 ICD cases, appropriate shocks were experienced in 28 cases (44\%), and inappropriate shocks in 19 cases (29\%). Additionally, data from the Japan Arrhythmia Device Industry Association obtained for overall device usage analysis revealed that CRTP was more commonly used in children than in adults.
\end{abstract}

Conclusions: There is an increasing need for pediatric device therapy, especially CRTP. However, many children's hospitals were still using DDD pacemakers in 2012. Although the demand for device therapy in children may be small, it is indispensable in pediatric cardiology. (Circ J 2014; 78: 1710-1716)

Key Words: Antiarrhythmic implantable device; Cardiac resynchronization therapy with a biventricular pacemaker (CRTP); Cardiac resynchronization therapy with a defibrillator (CRTD); Implantable cardioverter defibrillator (ICD); Pediatrics

$\mathbf{I}$ n Japan, implantable pacemakers are the most commonly used antiarrhythmic devices in children, although the use of implantable cardioverter defibrillators (ICDs) and cardiac resynchronization therapy with a biventricular pacemaker (CRTP) or a defibrillator (CRTD) is less common, and epidemiological studies have not been described. Furthermore, it has been common practice to use dual-chamber (DDD) pacemakers for CRT in children, because it is difficult for children's hospitals to meet the facility accreditation criteria for biventricular pacemaker implantation set by the Japanese Ministry of
Health, Labour and Welfare for the national insurance scheme. However, it is not well understood how common this practice actually is. The purpose of the present study therefore was to understand the use of ICDs, CRTP, CRT-DDD and CRTD in Japanese pediatric patients younger than 16 years of age.

\section{Editorial p 1579}

Received November 8, 2013; revised manuscript received March 12, 2014; accepted March 24, 2014; released online April 24 , 2014 Time for primary review: 24 days

Department of Pediatric Electrophysiology, Pediatric Medical Care Center, Osaka City General Hospital, Osaka (T.S.); Department of Pediatrics Cardiology, Saitama Medical University International Medical Hospital, Saitama (N.S.); Department of Pediatric Cardiology, Shizuoka Children's Hospital, Shizuoka (J.Y.); Department of Pediatric Cardiology, National Cerebral and Cardiovascular Center, Suita (A.M.); Department of Cardiovascular Surgery (K.H.), Department of Cardiology (H.U.), Fukuoka Children's Hospital, Fukuoka; and Department of Pediatric Cardiology, Nagano Children's Hospital, Nagano (S.Y.), Japan

Committee Members of Japanese Heart Rhythm Society, Device Implantation in Children

Mailing address: Tsugutoshi Suzuki, MD, Department of Pediatric Electrophysiology, Pediatric Medical Care Center, Osaka City General Hospital, 2-13-22 Miyakojima-hondori, Miyakojima-ku, Osaka 534-0021, Japan. E-mail: tsugutoshi@me.com

ISSN-1346-9843 doi:10.1253/circj.CJ-13-1379

All rights are reserved to the Japanese Circulation Society. For permissions, please e-mail: cj@j-circ.or.jp 


\begin{tabular}{|c|c|c|c|}
\hline & ICD & CRTP and CRT-DDD & CRTD \\
\hline No. of cases & 64 & 81 (47 with DDD, 58\%) & 7 \\
\hline Date of implantation & Oct 1999 - Nov 2012 & Nov 2001 - Nov 2012 & Nov 2007 - Aug 2011 \\
\hline Age at implantation (years) (range) & $12.6(0.3-15.5)$ & $3.5(0.2-15.5)$ & $13.1(1.8-15.3)$ \\
\hline Height (cm) (range) & $153(70-179)$ & $90(51-166)$ & $151(65-175)$ \\
\hline Weight (kg) (range) & $43.8(5.8-110)$ & $10.5(2.9-136)$ & $35(5.1-55)$ \\
\hline \multicolumn{4}{|l|}{ Approach, $n$} \\
\hline Transvenous & 45 & 4 & 4 \\
\hline Epicardial & 16 & 75 & 3 \\
\hline Both & 3 & 2 & 0 \\
\hline \multicolumn{4}{|l|}{ Shock lead/coil position, $n$} \\
\hline Right ventricle & 48 & $\mathrm{~N} / \mathrm{A}$ & 4 \\
\hline Subcutaneous, left & 5 & $\mathrm{~N} / \mathrm{A}$ & 0 \\
\hline Thoracic cavity, left & 3 & $\mathrm{~N} / \mathrm{A}$ & 0 \\
\hline Other & 8 & $\mathrm{~N} / \mathrm{A}$ & 3 \\
\hline \multicolumn{4}{|l|}{ Underlying cardiac condition, n } \\
\hline ARVC & 0 & 0 & 1 \\
\hline Asplenia & 0 & 6 & 0 \\
\hline Atrioventricular block, congenital & 0 & 11 & 0 \\
\hline CAVSD & 0 & 0 & 1 \\
\hline CPVT & 9 & 0 & 0 \\
\hline Dilated cardiomyopathy & 0 & 18 & 4 \\
\hline Double outlet right ventricle & 0 & 6 & 0 \\
\hline Hypertrophic cardiomyopathy & 17 & 0 & 0 \\
\hline Long QT syndrome & 17 & 0 & 0 \\
\hline Polysplenia & 0 & 7 & 0 \\
\hline Tetralogy of Fallot & 4 & 0 & 0 \\
\hline Transposition of the great arteries & 0 & 0 & 1 \\
\hline Ventricular tachycardia & 3 & 0 & 0 \\
\hline Other & 14 & 33 & 0 \\
\hline \multicolumn{4}{|l|}{ Shock delivery, n (\%) } \\
\hline Appropriate (+) & $28(44)$ & $\mathrm{N} / \mathrm{A}$ & 0 \\
\hline$(-)$ & $36(56)$ & $\mathrm{N} / \mathrm{A}$ & 1 \\
\hline Inappropriate (+) & $19(30)$ & $\mathrm{N} / \mathrm{A}$ & 0 \\
\hline$(-)$ & $45(70)$ & $\mathrm{N} / \mathrm{A}$ & 1 \\
\hline Not reported & 0 & $\mathrm{~N} / \mathrm{A}$ & 6 \\
\hline \multicolumn{4}{|l|}{ Outcome, n (\%) } \\
\hline Survival & $61(95)$ & - & - \\
\hline Death & $3(5)$ & - & - \\
\hline \multicolumn{4}{|l|}{ Cardiac outcome, n (\%) } \\
\hline Improved & $\mathrm{N} / \mathrm{A}$ & $67(83)$ & $3(43)$ \\
\hline Unchanged & $\mathrm{N} / \mathrm{A}$ & $6(7)$ & $1(14)$ \\
\hline Death after improvement & $\mathrm{N} / \mathrm{A}$ & $3(4)$ & 0 \\
\hline Death & $\mathrm{N} / \mathrm{A}$ & $5(6)$ & $1(14)$ \\
\hline Not reported & N/A & 0 & $2(29)$ \\
\hline
\end{tabular}

ARVC, arrhythmogenic right ventricular cardiomyopathy; CAVSD, complete atrioventricular septal defect; CPVT, catecholamine-induced polymorphic ventricular tachycardia; CRTP, cardiac resynchronization therapy with a biventricular pacemaker; CRT-DDD, cardiac resynchronization therapy with a DDD pacemaker; CRTD, cardiac resynchronization therapy with a defibrillator; ICD, implantable cardioverter defibrillator.

\section{Methods}

The study was designed by Japanese Heart Rhythm Society Committee Members of Device Implantation in Children. A questionnaire was sent to council member hospitals of the Japanese Society of Pediatric Cardiology and Cardiac Surgery to statistically analyze the use of ICD, CRT-DDD, CRTP and CRTD in pediatric ( $<16$ years old) patients up to 2012 . The questions included in the survey are listed in the Table.

Data were also obtained from the Japan Arrhythmia Device Industry Association (JADIA), which is an industry organization established by Japanese manufacturers and distributors of pacemakers, ICDs and CRT devices that collects all the information on implantable devices used across Japan. The numbers of pediatric cases of ICD, CRTP and CRTD use from 2000 to 2012 and adult cases of ICD, CRTP and CRTD use in 2012 
A

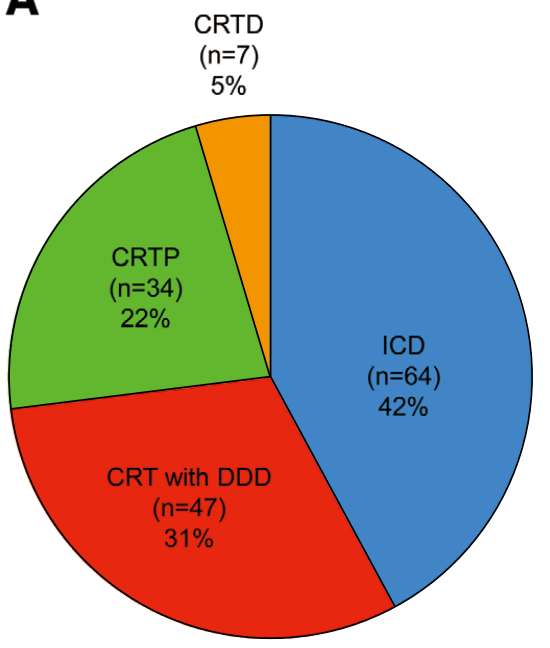

B

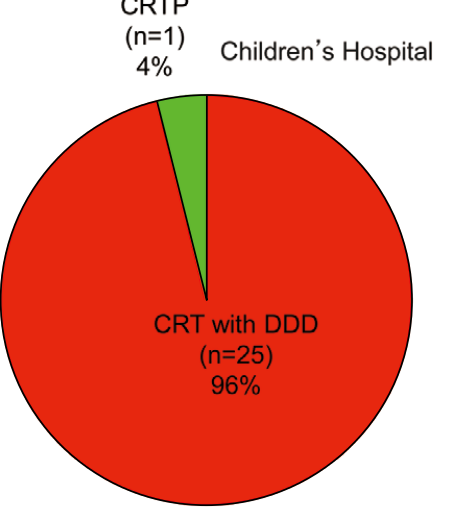

C

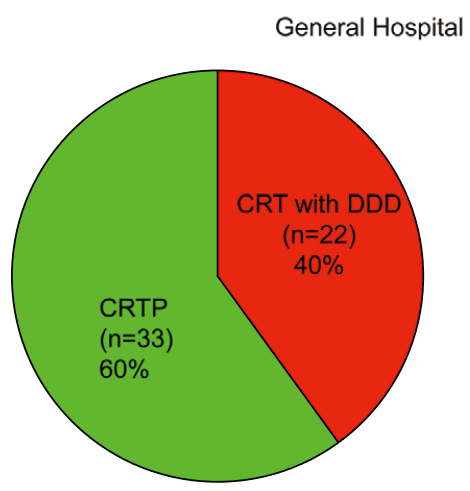

Figure 1. Pediatric use of ICD, CRTD, CRTP and CRT-DDD in Japan over the past 13 years. ICD, CRT-DDD, CRTP and CRTD were used in 64 (42\%), 47 (31\%), 34 (22\%) and 7 (5\%) cases, respectively, in the participating hospitals (A). CRT with a DDD pacemaker accounted for $96 \%$ in children's hospitals (B) vs. $41 \%$ in the general hospitals (C) of all cases of CRTP and CRT-DDD. CRTD, cardiac resynchronization therapy with a defibrillator; CRTP; cardiac resynchronization therapy with a biventricular pacemaker; ICD, implantable cardioverter defibrillator; DDD, dual-chamber.
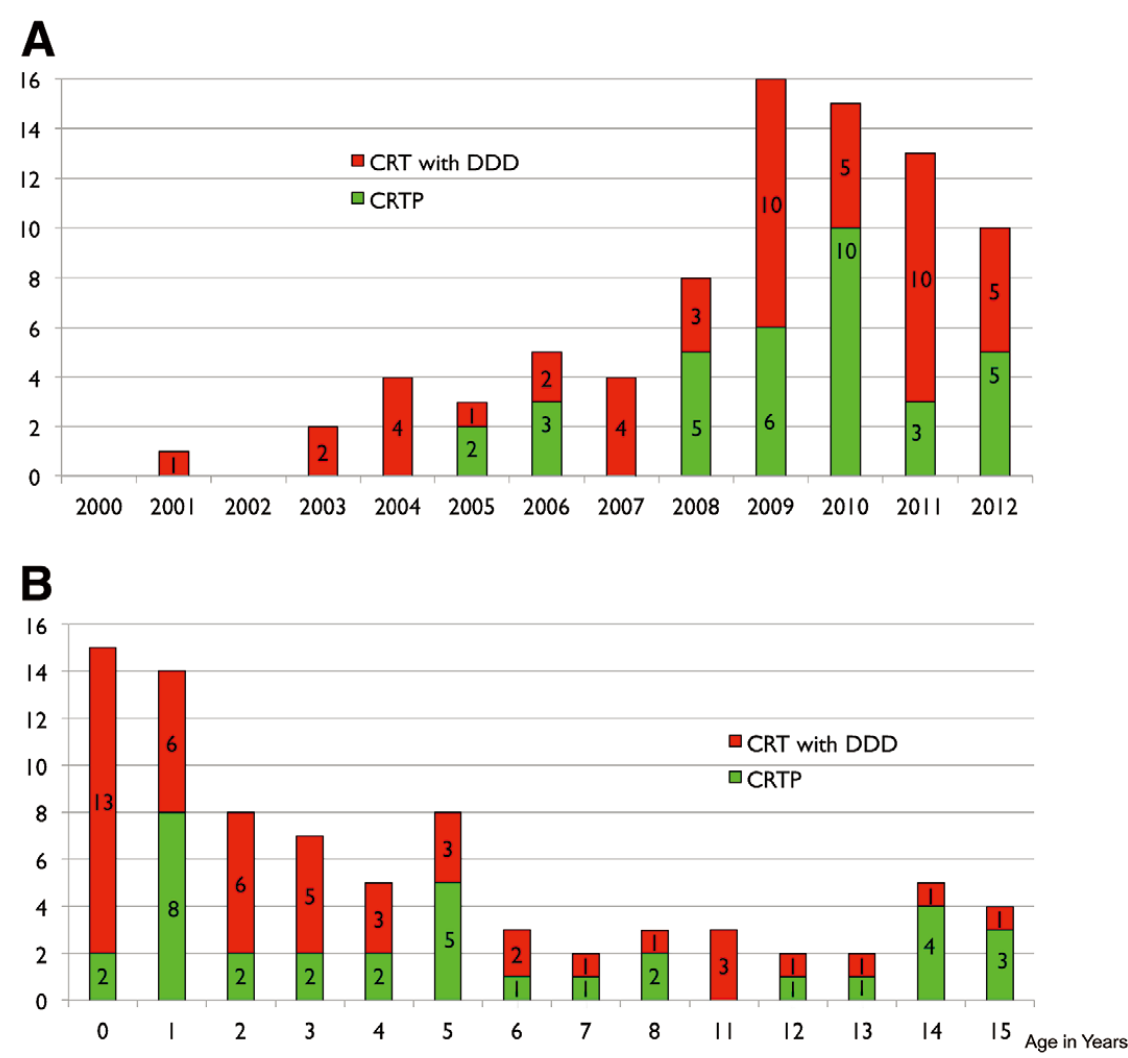

Figure 2. Use of cardiac resynchronization therapy with a biventricular pacemaker (CRTP) and that with a DDD pacemaker by year (A) and by age group (B). According to the questionnaire survey, DDD pacemakers were still used for CRT in nearly half of all cases of CRTP and CRT-DDD in recent years. By age, DDD pacemakers were used for CRT in all age groups, but their use was most notable in preschool children. CRTD, cardiac resynchronization therapy with a defibrillator; CRTP; cardiac resynchronization therapy with a biventricular pacemaker; ICD, implantable cardioverter defibrillator; DDD, dual-chamber. 
were obtained from JADIA. The number of CRT-DDD was not included in the JADIA data, and thus CRTP cases only represent those using biventricular CRTP devices. Pacemaker therapy using DDD or VVI was not counted in this study.

This study was conducted after review and approval by the ethics committee at Osaka City General Hospital.

Statistical analysis was performed by JMP software (SAS Institute, Cary, NC, USA).

\section{Results}

The questionnaire was sent to a total of 128 institutions, and 89 institutions $(70 \%)$ participated in the study. Pediatric device implantation was performed in 48 institutions (38\%), but not in 41 institutions (32\%). Among the responders were 15 children's hospitals (17\%) and 74 general hospitals (83\%). Among general hospitals, there were equal numbers of institutions that performed device implantation in children and those that did not. Of 15 children's hospitals, 10 (67\%) were performing device implantation indicating a higher need for antiarrhythmic device therapy compared with general hospitals.

The questionnaire responses are summarized in the Table. The most frequent reasons for device therapy included hypertrophic cardiomyopathy, long QT syndrome and catecholaminergic polymorphic ventricular tachycardia for ICD patients and dilated cardiomyopathy (DCM), cardiac failure because of right ventricular pacing in congenital complete atrioventricular block, polysplenia, asplenia, tetralogy of Fallot and atrioventricular septal defect for CRTP and CRT-DDD patients. DCM was the most common condition that required CRTD. A total of 152 patients received ICD $(n=64 ; 42 \%)$, CRT-DDD $(n=47 ; 31 \%)$, CRTP $(n=34 ; 22 \%)$ or CRTD $(n=7 ; 5 \%)$ between 1999 and 2012 (Figure 1A). The use of DDD pacemakers accounted for approximately $60 \%(n=47)$ of all CRTP and CRT-DDD cases $(\mathrm{n}=81)$. The percentage of CRT-DDD was much higher in children's hospital than in general hospitals [96\% (25/26) vs. $40 \%$ (22/55)] (Figures 1B,C). DDD was still commonly used for CRT in recent years accounting for nearly $50 \%$ of CRTP and CRT-DDD cases (Figure 2A), and DDD was more com-

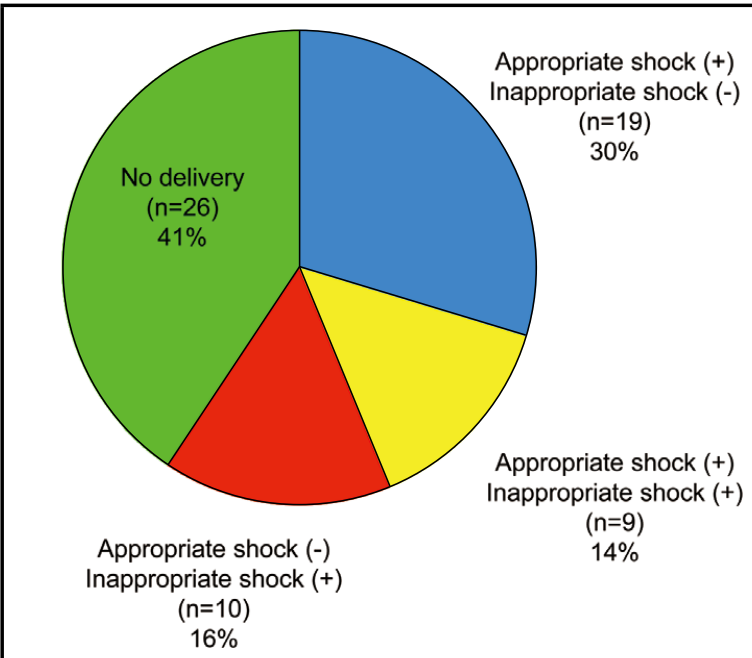

Figure 3. Outcomes after implantation of implantable cardioverter defibrillator (ICD), 19 (30\%) received appropriate shocks and never experienced inappropriate shocks, while 9 $(14 \%)$ experienced both appropriate and inappropriate shocks, and 10 (16\%) experienced inappropriate shocks but not appropriate shocks. Twenty-six children (41\%) experienced no shocks at all. Overall, about $1 / 3$ of ICD patients experienced inappropriate shocks.

monly used in preschool-age children though used in all ages (Figure 2B). Of 81 cases of CRT-DDD and CRTP, cardiac conditions improved in 67 cases $(83 \%)$, suggesting the effectiveness of CRT in pediatric cardiac failure. Overall, 8 cases of death were reported (Table), 5 of which occurred as a result of cardiac failure in children under 3 years of age with congenital heart disease.

Among 64 ICD patients, $19(30 \%)$ received appropriate shocks and never experienced inappropriate shocks, but 9 (14\%) experienced both appropriate and inappropriate shocks, and 10

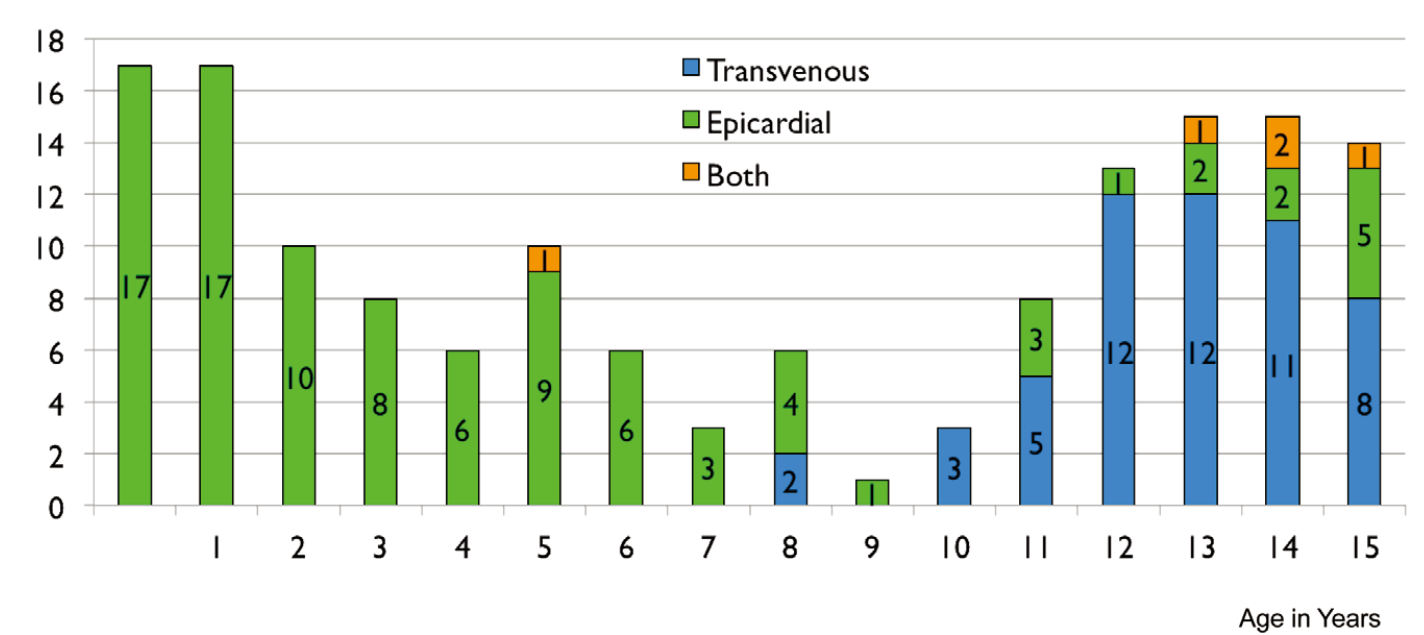

Figure 4. Surgical approaches used in pediatric device implantation by age. The use of the epicardial approach was most notable in children aged 9 years or younger. From 10 to 15 years of age, the transvenous approach was more common. (Data based on questionnaire survey results.) 


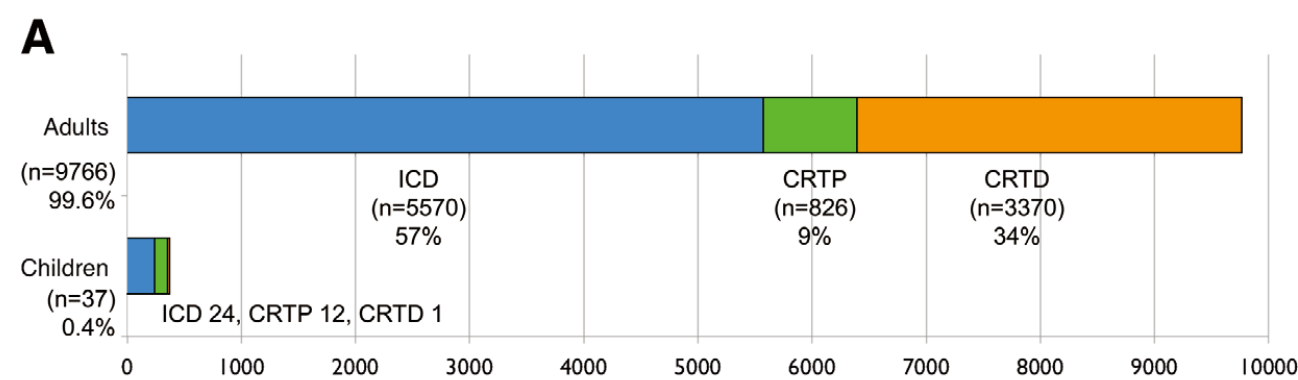

B

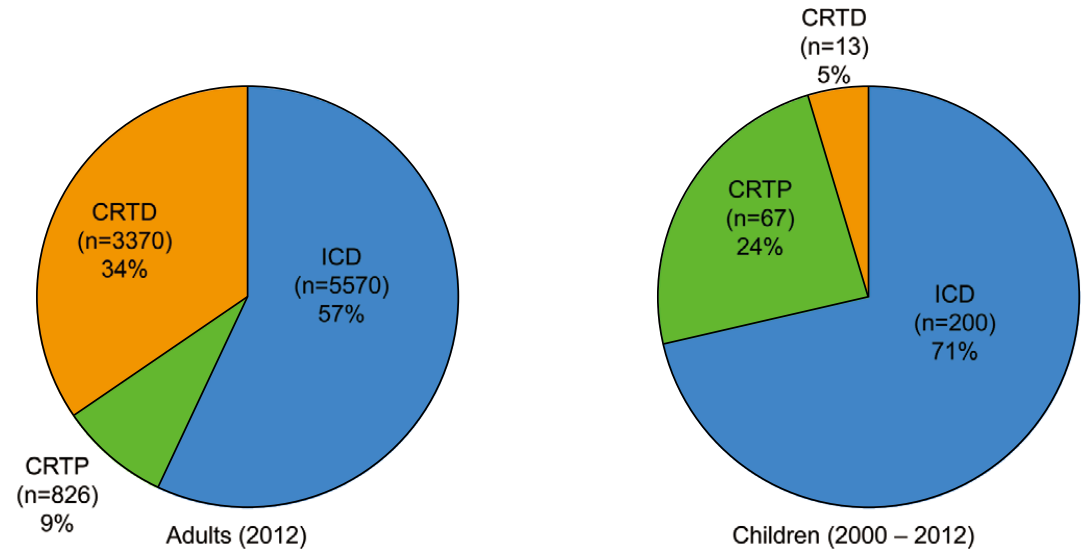

Figure 5. Summary of Japan Arrhythmia Device Industry Association (JADIA) data. (A) Number of devices implanted in adults and children ( $<16$ years of age) in 2012. Pediatric cases $(n=37)$ accounted for $0.4 \%$ of all cases. (B) Comparison of the type of device implanted in adult (2012) and pediatric patients (2000 to 2012). Note that CRTP was more common in children than in adults. CRTD, cardiac resynchronization therapy with a defibrillator; CRTP; cardiac resynchronization therapy with a biventricular pacemaker; ICD, implantable cardioverter defibrillator.

(16\%) experienced inappropriate shocks but not appropriate shocks (Figure 3); 26 children (41\%) experienced no shocks at all. Overall, approximately one-third of the pediatric population had experienced inappropriate shocks, and this rate was generally higher than that of the adult ICD patients. ${ }^{1}$

The surgical approaches are summarized. The epicardial approach was used in $62 \%(\mathrm{n}=94)$ of cases, the transvenous approach in $35 \%(n=53)$, and both approaches in $3 \%(n=5)$ (Table). The epicardial approach accounted for approximately two-thirds of the entire pediatric population and for almost all of the cases up to 9 years old (Figure 4). The epicardial approach was outnumbered by the transvenous approach after this age. For CRT, the epicardial approach was used in most cases, regardless of the child's age and bodyweight, whereas for ICD implantation the number of transvenous approaches increased after 8 years of age or over $30 \mathrm{~kg}$ of bodyweight (data not shown). As for ICD coil positions, epicardial placement accounted for approximately one-third of cases, and left subcutaneous leads were used in most of them (Table).

The data from the JADIA are summarized in Figure 5. In 2012, ICD, CRTP or CRTD device implantation was performed in 9,803 cases in total, and among them there were 37 pediatric cases ( $<16$ years of age), which accounted for $0.4 \%$ (Figure $5 \mathbf{A}$ ). Among those 37 cases, 24 received ICD, and 12 and 1 underwent CRTP and CRTD, respectively. Figure 5B shows a comparison of the types of devices implanted in adults (2012) and children (2000-2012). ICD implantation, CRTP and CRTD accounted for $57 \%, 9 \%$ and $34 \%$ of adult patients and $71 \%$, $24 \%$ and $5 \%$ of pediatric patients, respectively. It should be noted that CRTP was more frequently used in children compared with the adult population in which it was the least common therapy in 2012. The number of pediatric devices implanted increased over the past 13 years (Figure 6A); there were only 4 cases in 2000 , but by the 2010 s, 27 or more children were receiving an implantation every year with a maximum number of 40 in 2010. By age, pediatric device implantation was most frequently performed in children aged from 12 to 15 years (Figure 6B). A small peak was also seen from 0 to 5 years of age, indicating that implantable devices were also used in preschool children. ICDs accounted for approximately $80 \%$ of $12-$ to 15 -year-old patients, whereas CRTP was most common in preschool patients.

\section{Discussion}

This is the first epidemiological study to investigate the number of pacemakers and defibrillators implanted in the Japanese pediatric population under 16 years of age. Based on the results obtained, an estimated 27-40 children received ICD, CRTP or CRTD annually in the 2010s, and the trend in Japanese pediatric device therapy is best characterized by increased use of CRTP in preschool children and use of DDD devices for CRT, 


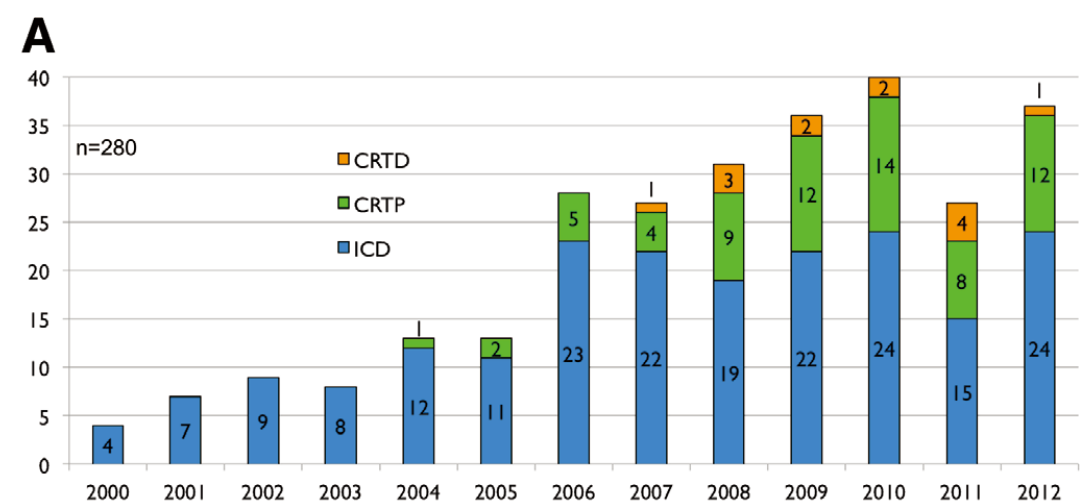

B

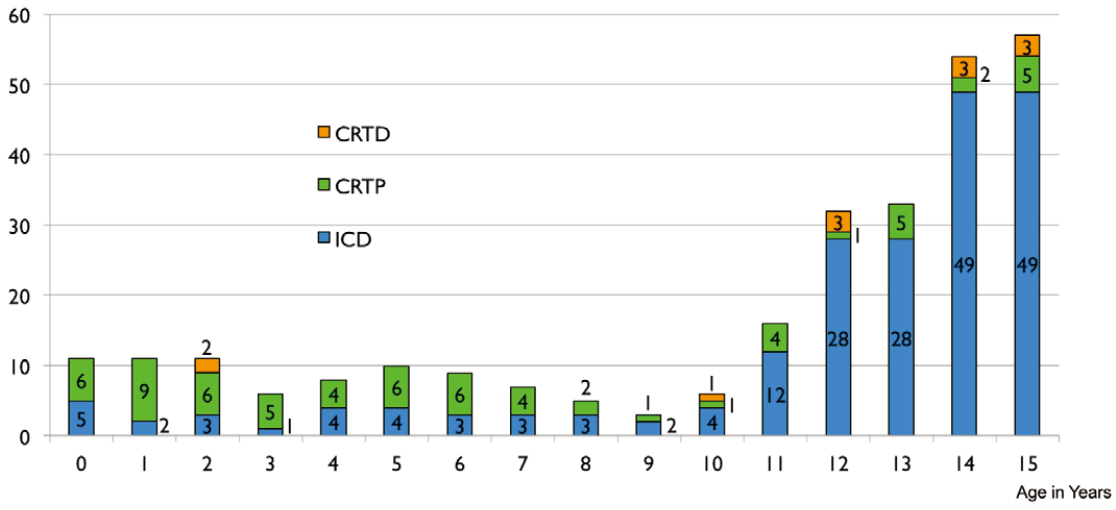

Figure 6. The 13-year trend in cardiac resynchronization therapy with a defibrillator (CRTD), CRT with a biventricular pacemaker (CRTP) and implantable cardioverter defibrillator (ICD) implantation in Japanese pediatric patients under 16 years of age. (A) ICDs were the only device used until 2003. Thereafter, the total number of devices implanted increased gradually up to 24 cases of ICD, 14 cases of CRTP and 2 cases of CRTD in 2010s. (B) Number of pediatric cases of CRTD, CRTP and ICD implanted by age. There were 2 peaks, a small peak at 0-5 years of age and a larger peak at 12-15 years of age. The former peak indicates that antiarrhythmic device implantation, primarily CRTP, was also performed in preschool-age children, while ICDs accounted for approximately $80 \%$ of the latter group. The data were obtained from the Japan Arrhythmia Device Industry Association and include all the pediatric cases from 2000 to $2012(n=280)$.

especially in children's hospitals.

The use of DDD devices for CRT is an important issue inherent to children's hospitals. In adults, DDD pacemakers were used for CRT before biventricular CRTP devices became available, ${ }^{2}$ but are no longer used. They were also conventionally used in children experiencing severe cardiac failure and dyssynchrony after surgical repair of congenital heart disease. ${ }^{3}$ The strongest advantage of CRTP devices over CRT using a DDD pacemaker is that the former is capable of programming the interventricular delay (beneficial for interventricular dyssynchrony) as well as unipolar pacing (may be used if the pacing threshold is increased after device implantation). In CRT with a DDD pacemaker, implantation of the cathode lead in the left ventricle and the anode lead in the right ventricle can worsen cardiac function because of anodal capture, which may result in single, right ventricular pacing. ${ }^{6,7}$ Based on the results of this study, however, DDD pacemakers were still used for CRT in 5-10 pediatric cases per year in the 2010 s, and most of these cases were reported from children's hospitals.

One of the main reasons that CRT-DDD is being used for children now is that the CRTP generator is too large to implant in small children and, unlike general hospitals, children's hospitals have no choice but to use DDD pacemakers for CRT, because these facilities are not generally accredited for biventricular CRTP devices according to the Japanese government's strict criteria for accreditation and thus CRTP devices are not covered by the national health insurance scheme. Given the promising role of CRT in congenital heart disease, ${ }^{4}$ coupled with the $83 \%$ improvement rate in cardiac condition after CRTP and CRT-DDD (Table) and the increasing demand for pediatric CRT especially in preschool-age children (Figures 2B,6), it is vital that CRTP devices are available for children. Regulatory changes to allow the use of these devices in children's hospitals, as commented by the responders to the questionnaire in the present study, are necessary.

In adult CRTP, transvenous intracardiac lead placement is commonly performed. In the present study, however, the epicardial approach was more frequently used. Although this was not an unexpected result for preschool children, it was also commonly used for older children, probably because access through the coronary sinus is often difficult in patients with congenital heart disease. As for ICD coil positions, epicardial placement accounted for approximately one-third of cases, and endocardial defibrillator leads were used in most of those for which various placement approaches were used in a trial-and-error manner. Radbill et al compared 78 transvenous and 39 epicar- 
dial (nontransvenous) ICD implantation cases and reported that the patients in the nontransvenous group were younger and had a smaller body size, shorter survival of ICD systems and poorer life prognosis than the transvenous group patients. ${ }^{5}$ Nonetheless, the epicardial approach remains the only option for many pediatric patients. More studies are needed to establish a safer and more reliable approach for this subset of patients.

Sudden cardiac death may occur a long time after congenital heart disease surgery, ${ }^{8}$ and the efficacy of ICD in preventing such deaths has been reported. ${ }^{9,10}$ Cuo et al reported that echocardiogram is very effective in determining whether CRT is indicated and in selecting the position of CRT in patients with a single ventricle. ${ }^{11}$ The need for pediatric ICD implantation, CRTP and CRTD is still small in Japan, but efficacy of these devices has already been reported in other countries, ${ }^{4}$ regardless of the patient's ethnic background. ${ }^{12,13}$ To meet the potential increase in the demand for device therapy in Japan, these devices must be made available to children's hospitals as well.

\section{Conclusions}

Based on the results of the present study, the overall demand for ICD, CRTP and CRTD is estimated to be from 27 to 40 cases per year in the $2010 \mathrm{~s}$ in the Japanese pediatric population. The current government accreditation criteria for CRTP device are imposing a challenge to children's hospitals. Although the need is small, the device is essential for the pediatric population. Prompt action is recommended to solve this problem.

\section{Acknowledgments}

We are grateful to Dr Atsuhito Takeda, Hokkaido University Hospital; Dr Aya Miyazaki, National Cerebral and Cardiovascular Center Hospital; Dr Eiki Nishihara, Ogaki Municipal Hospital; Dr Fukiko Ichida, Toyama University Hospital; Dr Hideshi Tomita, Showa University Northern Yokohama Hospital; Dr Hidetake Tejima, National Hospital Organization Nagasaki Medical Center; Dr Hiraku Doi, Kyoto University Hospital; Dr Hirohiko Shiraishi, Jichi Medical University Hospital; Dr Hiroki Kajino, Asahikawa Medical University; Dr Hiroshi Suzuki, Niigata University Medical \& Dental Hospital; Dr Hiroya Ushinohama, Fukuoka Children's Hospital \& Medical Center for Infectious Diseases; Dr Isao Takeuchi, Japanese Red Cross Medical Center; Dr Jun Yoshimoto, Shizuoka Children's Hospital; Dr Junji Fukuhara, Nihon University Itabashi Hospital; Dr Junpei Soumura, Shiga University of Medical Science Hospital; Dr Kanta Kishi, Osaka Medical College Hospital; Dr Kazuhiro Takahashi, Okinawa Children's Medical Center; Dr Keiko Toyohara, Tokyo Women's Medical University Hospital; Dr Kentarou Asou, St. Marianna University School of Medicine; Dr Koichiro Niwa, St. Luke's International Hospital; Dr Kozo Matsuo, Chiba Cardiovascular Center; Dr Mari Iwamoto, Yokohama City University; Dr Masahide Kaneko, National Center for Child Health and Development; Dr Masahiko Kishiro, Juntendo University Hospital; Dr Masahiko Matsumura, Tenri Hospital; Dr Masaru Miura, Tokyo Metropolitan Children's Medical Center; Dr Masaru Yamakawa, Kobe City Medical Center General Hospital; Dr Masatoshi Shimada, Nagano Children's Hospital; Dr Motoki Takamuro, Hokkaido Medical Center for Child Health and Rehabilitation; Dr Naoki Ohashi, Social Insurance Chukyo Hospital; Dr Naomi Nakagawa, Hiroshima City Hospital; Dr Shigemitsu Iwai, Osaka Medical Center and Research Institute for
Maternal and Child Health; Dr Shigeru Uemura, Showa University Northern Yokohama Hospital; Dr Shintarou Kishimoto, Kurume University; Dr Shouji Suzuki, University of Yamanashi Hospital; Dr Shunji Uchita, Wakayama Medical University; Dr Takaomi Minami, Jichi Children’s Medical Center Tochigi; Dr Takashi Honda, Kitasato University Hospital; Dr Takuro Ohno, Oita Prefectural Hospital; Dr Tomoaki Murakami, Chiba Children's Hospital; Dr Tomomi Nishimura, Sakakibara Memorial Hospital; Dr Toshihide Asou, Kanagawa Children's Medical Center; Dr Toshiki Kobayashi, Saitama Medical University International Medical Center; Dr Yasunobu Hayabuchi, Tokushima University Hospital; Dr Yoshiaki Kato, University of Tsukuba Hospital; Dr Yoshifumi Okano, Toho University Omori Medical Center; and Dr Yusuke Iwata, Gifu Prefectural General Medical Center, for their contributions to the survey.

\section{Disclosures}

Financial Support: None.

\section{References}

1. van Rees JB, Borleffs CJW, de Bie MK, Stijnen T, van Erven L, Bax $\mathrm{JJ}$, et al. Inappropriate implantable cardioverter-defibrillator shocks. J Am Coll Cardiol 2011; 57: 556-562.

2. Alonso C, Leclercq C, Victor F, Mansour H, de Place C, Pavin D, et al. Electrocardiographic predictive factors of long-term clinical improvement with multisite biventricular pacing in advanced heart failure. Am J Cardiol 1999; 84: 1417-1421.

3. Buckingham TA, Candinas R, Duru F, Pagotto E, Schönbeck M, Amann FW, et al. Acute hemodynamic effects of alternate and combined site pacing in patients after cardiac surgery. Pacing Clin Electrophysiol 1999; 22: 887-893.

4. van der Hulst AE, Delgado V, Blom NA, van de Veire NR, Schalij $\mathrm{MJ}$, Bax JJ, et al. Cardiac resynchronization therapy in paediatric and congenital heart disease patients. Eur Heart J 2011; 32: 2236-2246.

5. Radbill AE, Triedman JK, Berul CI, Fynn-Thompson F, Atallah J, Alexander ME, et al. System survival of nontransvenous implantable cardioverter-defibrillators compared to transvenous implantable cardioverter-defibrillators in pediatric and congenital heart disease patients. Heart Rhythm 2010; 7: 193-198.

6. Dendy KF, Powell BD, Cha YM, Espinosa RE, Friedman PA, Rea RF, et al. Anodal stimulation: An underrecognized cause of nonresponders to cardiac resynchronization therapy. Indian Pacing Electrophysiol J 2011; 11: 64-72.

7. Swerdlow CD, Friedman PA. Advanced ICD troubleshooting: Part II. Pacing Clin Electrophysiol 2006; 29: 70-96.

8. Mizuno A, Niwa K, Matsuo K, Kawada M, Miyazaki A, Mori Y, et al. Survey of reoperation indications in tetralogy of Fallot in Japan. Circ J 2013; 77: 2942-2947.

9. Walsh EP, Cecchin F. Arrhythmias in adult patients with congenital heart disease. Circulation 2007; 115: 534-545.

10. Berul CI, Van Hare GF, Kertesz NJ, Dubin AM, Cecchin F, Collins $\mathrm{KK}$, et al. Results of a multicenter retrospective implantable cardioverter-defibrillator registry of pediatric and congenital heart disease patients. J Am Coll Cardiol 2008; 51: 1685-1691.

11. Cua CL, Feltes TF. Echocardiographic evaluation of the single right ventricle in congenital heart disease: Results of new techniques. Circ J 2012; 76: $22-31$.

12. Momomura S, Tsutsui H, Sugawara Y, Ito M, Mitsuhashi T, Fukamizu $\mathrm{S}$, et al; MOMIJI Study Investigators. Clinical efficacy of cardiac resynchronization therapy with an implantable defibrillator in a Japanese population: Results of the MIRACLE-ICD outcome measured in Japanese indication (MOMIJI) study. Circ J 2012; 76: 1911-1919.

13. Chan $\mathrm{CP}, \mathrm{Yu} \mathrm{CM}$. Cardiac resynchronization therapy: Is there any racial difference? Circ J 2012; 76: 1830-1831. 